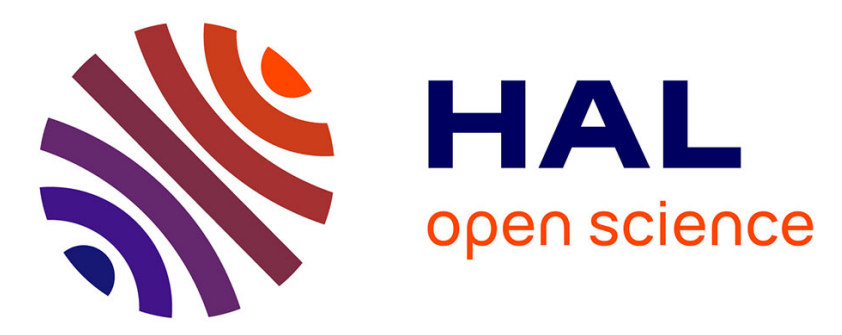

\title{
Axial plane dissimilarities of two identical Lenke-type 6C scoliosis cases visualized and analyzed by vertebral vectors
}

Tamás Illés, Máté Burkus, Szabolcs Somoskeöy, Fabien Lauer, François Lavaste, Jean Dubousset

\section{To cite this version:}

Tamás Illés, Máté Burkus, Szabolcs Somoskeöy, Fabien Lauer, François Lavaste, et al.. Axial plane dissimilarities of two identical Lenke-type $6 \mathrm{C}$ scoliosis cases visualized and analyzed by vertebral vectors. European Spine Journal, 2018, 27 (9), pp.2120-2129. 10.1007/s00586-018-5577-1 . hal02060150

\author{
HAL Id: hal-02060150 \\ https://hal.science/hal-02060150
}

Submitted on 21 Feb 2020

HAL is a multi-disciplinary open access archive for the deposit and dissemination of scientific research documents, whether they are published or not. The documents may come from teaching and research institutions in France or abroad, or from public or private research centers.
L'archive ouverte pluridisciplinaire HAL, est destinée au dépôt et à la diffusion de documents scientifiques de niveau recherche, publiés ou non, émanant des établissements d'enseignement et de recherche français ou étrangers, des laboratoires publics ou privés. 


\title{
Axial plane dissimilarities of two identical Lenke-type $6 \mathrm{C}$ scoliosis cases visualized and analyzed by vertebral vectors
}

\author{
Tamás S. Illés ${ }^{1,2,7} \cdot$ Máté Burkus $^{3} \cdot$ Szabolcs Somoskeőy $^{4} \cdot$ Fabien Lauer $^{5} \cdot$ Francois Lavaste $^{6} \cdot$ Jean F. Dubousset $^{7}$
}

\begin{abstract}
Purpose The global appearance of scoliosis in the horizontal plane is not really known. Therefore, the aims of this study were to analyze scoliosis in the horizontal plane using vertebral vectors in two patients classified with the same Lenke group, and to highlight the importance of the information obtained from these vertebral vector-based top-view images in clinical practice. Methods Two identical cases of scoliosis were selected, based on preoperative full-body standing anteroposterior and lateral radiographs obtained by the EOS ${ }^{\mathrm{TM}} 2 \mathrm{D} / 3 \mathrm{D}$ system. Three-dimensional (3D) surface reconstructions of the spinal curves were performed by using sterEOS ${ }^{\mathrm{TM}} 3 \mathrm{D}$ software before and after surgery. In both patients, we also determined the vertebral vectors and horizontal plane coordinates for analyzing the curves mathematically before and after surgery.

Results Despite the identical appearance of spinal curves in the frontal and sagittal planes, the horizontal views seemed to be significantly different. The vertebral vectors in the horizontal plane provided different types of parameters regarding scoliosis and the impact of surgical treatment: reducing lateral deviations, achieving harmony of the curves in the sagittal plane, and reducing rotations in the horizontal plane.

Conclusions Vertebral vectors allow the evolution of scoliosis curve projections in the horizontal plane before and after surgical treatment, along with representation of the entire spine. The top view in the horizontal plane is essential to completely evaluate the scoliosis curves, because, despite the similar representations in the frontal and sagittal planes, the occurrence of scoliosis in the horizontal plane can be completely different.
\end{abstract}

Keywords Scoliosis $\cdot$ Vertebral vector $\cdot$ Horizontal plane $\cdot$ Top view $\cdot$ EOS ${ }^{\text {TM }}$ 2D/3D

\section{Introduction}

Scoliosis is not a simple lateral deviation, but rather a complex three-dimensional (3D) deformity of the spine. Besides the clearly visible frontal and sagittal curves, deformations (vertebral rotation and torsion) in the horizontal plane are 
also present. As a result of this complex mechanism, the spine and entire trunk deviate from its normal, mid-sagittal plane of symmetry. The diagnosis and classification of scoliosis are almost exclusively based on frontal and lateral (LAT) radiographs, as rotational changes in the horizontal plane are ignored. Deviation of the entire trunk from its normal mid-sagittal plane of symmetry is also overlooked [1].

Several attempts have been made to visualize and to describe the real 3D appearance of spinal deformity [2, 3], with particular attention to axial plane lesions $[4,5]$, but the application of modalities has only slightly improved the limitations of the 3D display [6-8]. A recently proposed method is the concept of the plane of maximum curvature (PMC) and its visualization through DaVinci representation. The PMC is constructed by using the centroids of the two end vertebrae and the apical vertebra of a curve. The PMC is analogous to the term "plan d'élection", which was introduced by Stagnara et al. [9] to evaluate the significant scoliotic curves radiologically. By definition, the PMC is an abstract, continually changing, extraspinal auxiliary plane and as stated by Stokes, "not a true 3D visualization, but more of a 'quasi-3D rendering' and as such, it is difficult to understand, laborious to reproduce, and explain its clinical significance of daily practice [10].

The most accurate assessment of alterations in the axial plane seems to be achieved by computed tomography (CT). However, its usefulness is limited in the routine diagnosis of scoliosis, because only a short spinal segment can be examined; moreover, it is accompanied by significant radiation exposure. The other problem is the effect of the supine position during imaging, which may lead to significant alterations of the curves in the coronal, sagittal, and, consequently, horizontal planes [11].

In the absence of overhead view visualization, the efforts of 3D classifications have not been successful either. The most frequently used Lenke classification has been introduced as "a prelude to 3D classification". It was proposed to be comprehensive and all-inclusive for all curve types, objective, and easy to use, aiding in standardized treatment with direct applicability in routine clinical practice. As a new feature, it emphasizes the consideration of sagittal spinal alignment but disregard changes in the horizontal plane, namely the vertebral rotation and translation of the spine [12].

The concept of vertebral vectors has been introduced by our workgroup to visualize and evaluate spinal deformities in a truly 3D manner. A vertebral vector is, by its definition, a replacement for a real vertebra while preserving crucial information for its size, position, orientation, and rotation in $3 \mathrm{D}$ projection. The starting points of the vertebral vectors are in the middle of an interpedicular segment, and they run parallel to the upper endplate, ending in the level where this parallel line intersects the segment joining the two anterior points of the vertebral endplates, defining the posteroanterior axis of the vertebra. It is possible to define the coordinates of these two points, thereby depicting the posteroanterior vertebral axis (vertebral vector) in an individually calibrated coordinate system, also described by us [13]. This method, which represents the full spine, provides simple, well-established mathematical ways to evaluate the curvatures in the frontal, sagittal, and horizontal planes.

The aims of this study were to analyze scoliosis in the horizontal plane in two patients classified with the same Lenke group, and to emphasize the importance of the awareness of the appearance of scoliosis in the horizontal plane before considering its classification. To our knowledge, the analysis of two patients with scoliosis classified into the same Lenke group and displayed by vertebral vectors in the overhead view has not been made yet.

\section{Materials and methods}

Two patients with scoliosis classified, according to the Lenke criteria, into the same group with the same lumbar and sagittal modifiers by three independent, experienced scoliosis experts [8] were chosen for the analysis of their preoperative and postoperative appearance in the horizontal plane. These two cases were selected from 814 retrospectively analyzed full-body standing anteroposterior (AP) and LAT radiographs obtained by the EOSTM $2 \mathrm{D} / 3 \mathrm{D}$ system (EOS Imaging, Paris, France). This X-ray machine is capable of capturing full-body standing orthogonal AP and LAT radiographs simultaneously, allowing an accurate and realistic 3D reconstruction of the skeletal system, including the spine [14]. Digital images were stored in the institutional Picture Archiving and Communication System network (Aspyra AccessNet v6.2, Aspyra Inc., Westlake Village, CA, USA).

Surface 3D reconstructions of vertebrae of the entire spine were performed using sterEOS ${ }^{\mathrm{TM}} 3 \mathrm{D}$ workstation software version 1.3.4.3740 (EOS Imaging) after performing the full 3D procedure using AP and LAT EOSTM $2 \mathrm{D}$ images. The completed sterEOS ${ }^{\mathrm{TM}} 3 \mathrm{D}$ reconstructions were used for vertebral vector generation.

To facilitate the understanding of the 3D images, simplify visualization of the entire spine, especially in the horizontal plane, and create the possibility of mathematical characterization, we introduced the concept of vertebral vectors [13]. The vector is a mathematical entity characterized by its initial and terminal points by its length and spatial direction, commonly represented by a directed line segment.

The initial point of the vertebral vector is the middle point of the interpedicular line connecting the two pedicular centroids. By definition, the vertebral vector is parallel with the upper endplate of the vertebra. Therefore, the terminal point is situated on the ventral surface of the vertebral body when 
the anterior wall is dissected by the vector at the level of the pedicular centroids. The vertebral vector length is proportional to the size of the vertebral body, and it determines the posteroanterior axis of the vertebra (Fig. 1a-c).

An individually calibrated coordinate system was created to determine the vertebral vector coordinates. The $x$-axis was the straight line connecting the two centers of the acetabulum. The $y$-axis was perpendicular to the $x$-axis, and it passed through the midpoint of the interacetabular length, whose axis is located in the median sagittal plane of the body. The $z$-axis passes through the same point and perpendicular to both the previous axes.
An individually characteristic scale was created by dividing the interacetabular distances by 200 units, and this was used as the base unit on each axis of the coordinate system (Fig. 1d).

The vertebral vector generation software of MATLAB (MathWorks Inc., Natick, MA, USA) was used to directly acquire $3 \mathrm{D}$ coordinate data of vertebral vector-specific elements stored in sterEOS ${ }^{\mathrm{TM}}$ 3D models, as well as to visualize generated vertebral vectors in the three planes. Vertebral vector-associated parameters-coordinates $x, y$, and $z$ for the left and right pedicular centroids, initial point $\mathrm{A}$, and terminal point $B$ of the vertebral vectors-were recorded.

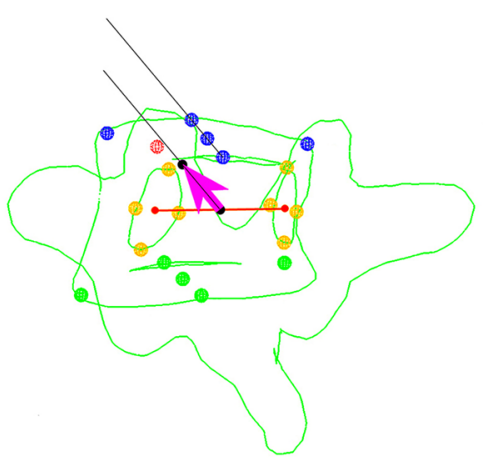

A

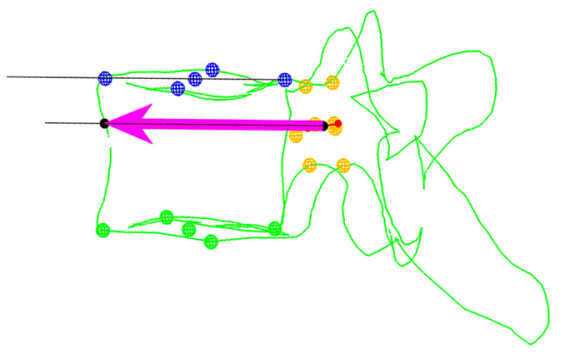

B

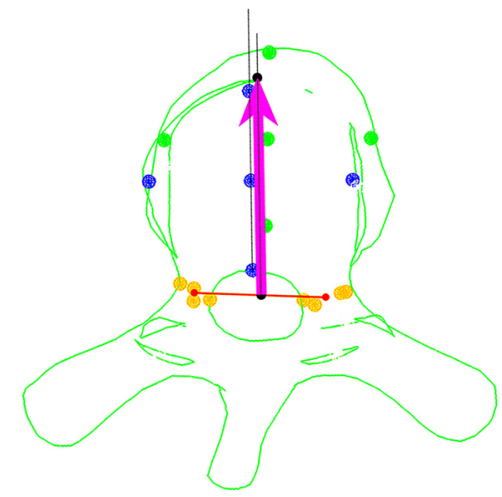

C

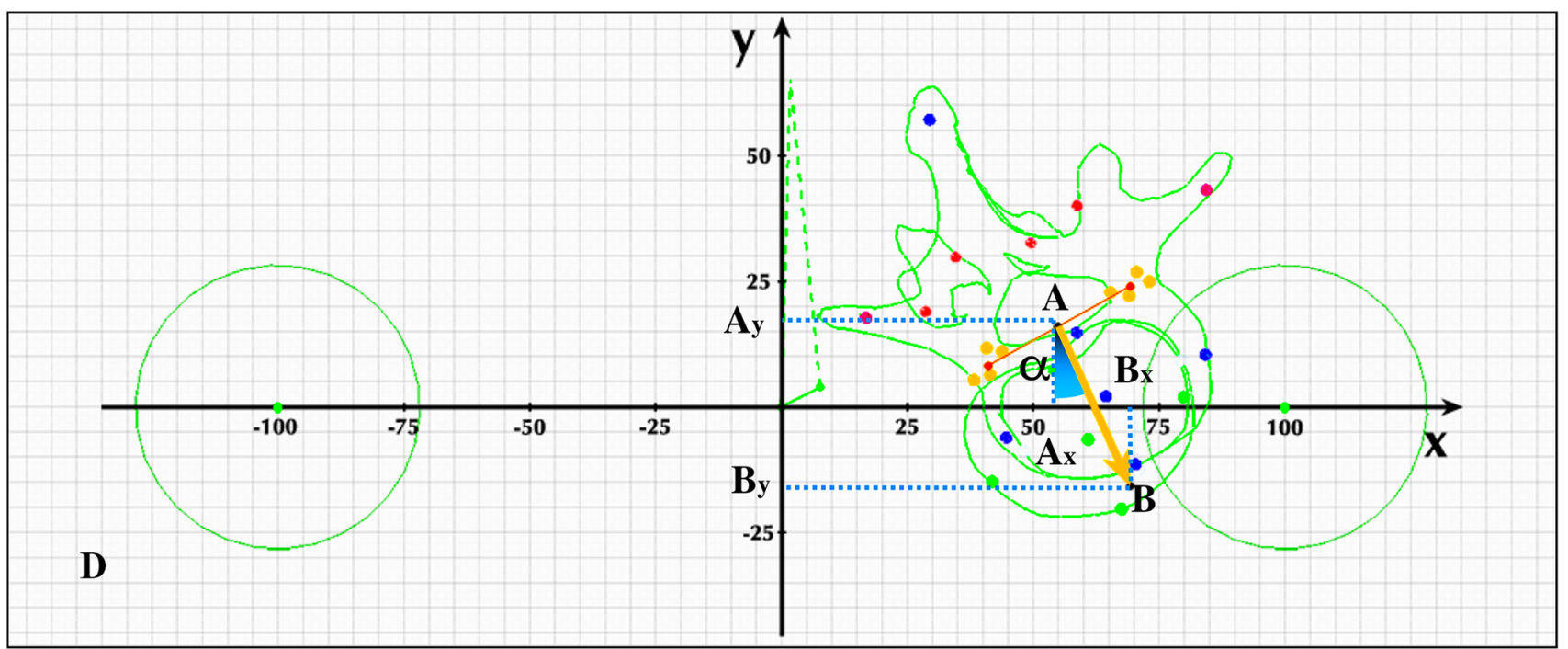

Fig. 1 Determination of the vertebral vector and individual coordinate system. Vertebral vector views in the coronal plane: the initial point A (black dot) of the vector is the midpoint of the interpedicular line (orange) connecting the two pedicular centroids (a). Sagittal plane: the vertebral vector is parallel to the upper endplate of the vertebra (b). The terminal point of the vector is determined by the intersection of this line and the ventral surface of the vertebral body. Horizontal plane: the vertebral vector defines the posteroanterior axis of the vertebral body (c). Vertebral vector in the coordinate system (d). The $x$-axis in this coordinate system is the interacetabular axis connecting the two acetabular centers. The $y$-axis is the sagittal median axis of the body, which is perpendicular to the $x$-axis in the midpoint of the interacetabular distance. The $z$-axis is perpendicular to the $x$-axis and $y$-axis in the midpoint of the interacetabular distance (not visible in this view). The scale of the coordinate system is individualized, because, regardless of the real length of the interacetabular distance, it has always been divided into 200 units. Thus, the scale unit is $50 \%$ of the interacetabular distance/100, and this base unit is used for each axis of the coordinate system. In this coordinate system, it is possible to determine each vector point in all three planes using basic vector algebra 
In this study, we focused only on the vertebral vector projection in the horizontal plane. Thus, we only used the $x$ and $y$ parameters of the start and end points $\left(\mathrm{A}_{x}, \mathrm{~A}_{y}, \mathrm{~B}_{x}\right.$, and $\left.\mathrm{B}_{y}\right)$ of the vertebral vectors.

These coordinates are used directly to determine lateral translation $A_{x}$ and $B_{x}$ from the $y$-axis. The vector angle $\alpha$ relative to the $y$-axis was calculated using the trigonometry tangent function (Fig. 1d):

$\operatorname{tg} a=\frac{\left(B_{x}-A_{x}\right)}{\left(B_{y}-A_{y}\right)}$

After measuring the absolute value of the interacetabular distance in mm using the sterEOS ${ }^{\mathrm{TM}} 3 \mathrm{D}$ software, the following formulas were used to determine the real distances $(d)$ in mm of the starting and endpoints of the vertebral vectors from the $x$-axis $(\mathrm{d} x)$ and $y$-axis $(\mathrm{d} y)$ :

\section{Results}

After analyzing the preoperative frontal and sagittal radiographs, both cases of scoliosis were classified as Lenke group 6 by the three examiners. The lumbar modifier was determined in both cases as $\mathrm{C}$, because the apical vertebrae fully lie off the center sacral vertical lines. Sagittal thoracic modifiers were classified minus (-) (hypokyphosis), because the thoracic kyphosis was less than $10^{\circ}$ in both cases. Therefore, both curves were classified in the same Lenke class, 6/C-, without knowing the horizontal appearance.

The $3 \mathrm{D}$ reconstruction of the entire spine of the first and second cases of scoliosis is demonstrated in Figs. $2 \mathrm{a}-\mathrm{d}$ and $3 \mathrm{a}-\mathrm{d}$, respectively, to show the similarity of the two scoliotic curves in the frontal and sagittal planes. Both patients were surgically treated based on the Cotrel-Dubousset philosophy and the axial rod rotation maneuver of the prebent rod.

$\mathrm{d} x=A_{x} \frac{\text { interacetabular distance }(\mathrm{mm})}{200} ; \mathrm{d} y=A_{y} \frac{\text { interacetabular distance }(\mathrm{mm})}{200}$.

After visualizing the preoperative 3D top-view images (Fig. 4a, b), differences between the two curves were

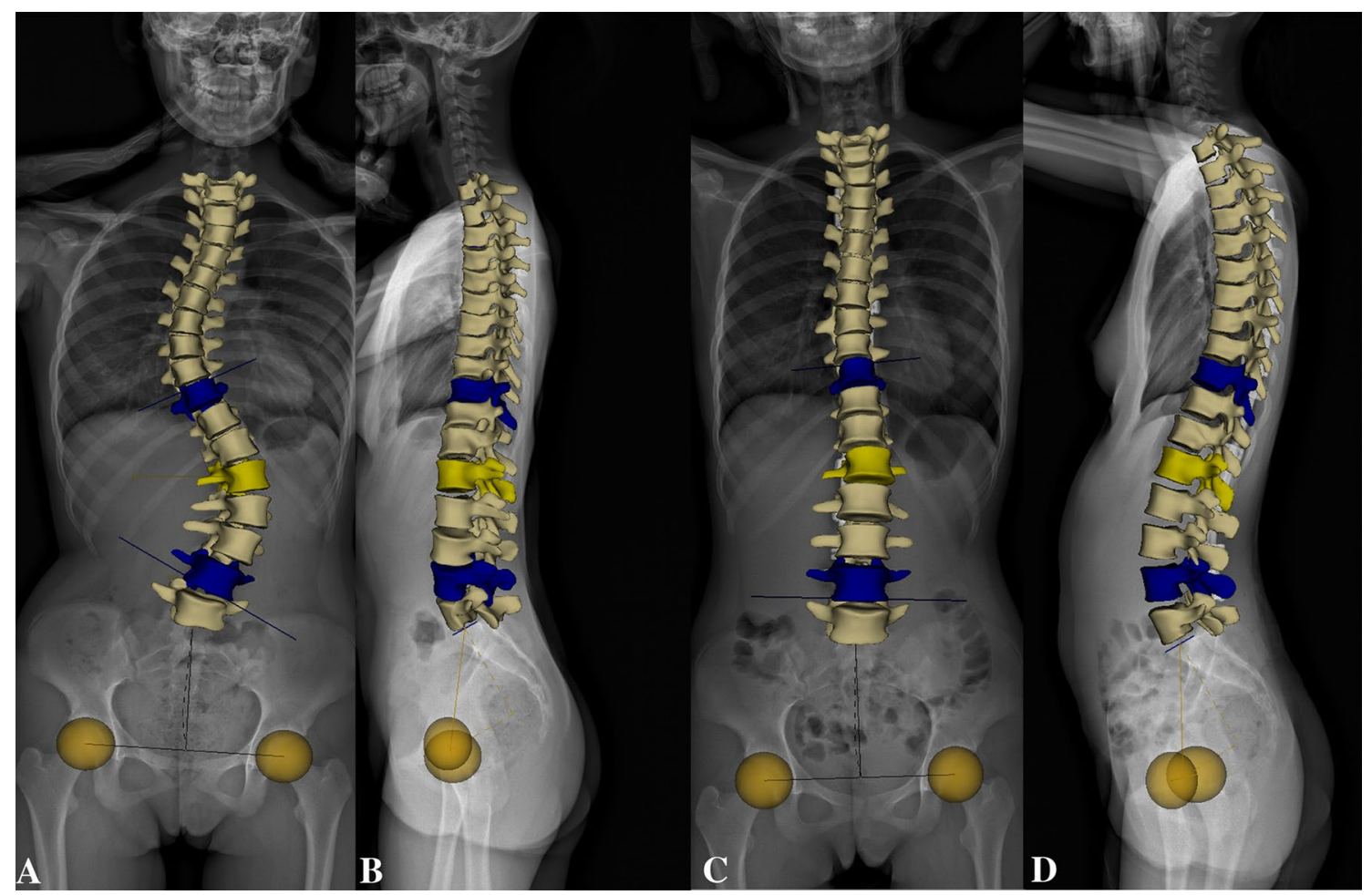

Fig. 2 First case of scoliosis classified as Lenke 6/C. $48^{\circ}$ right structural thoracic and $55^{\circ}$ left main lumbar curves (a). The thoracic kyphosis between the T4 and T12 vertebrae is $5^{\circ}$, whereas the lumbar lordosis measured between the L1 and L5 vertebrae is $32^{\circ}$ (b). Post- operative frontal curves are $8^{\circ}$ in the thoracic area and $4^{\circ}$ in the lumbar area (c). Both sagittal curves increase postoperatively (thoracic kyphosis $33^{\circ}$; lumbar lordosis $46^{\circ}$ ) (d) 


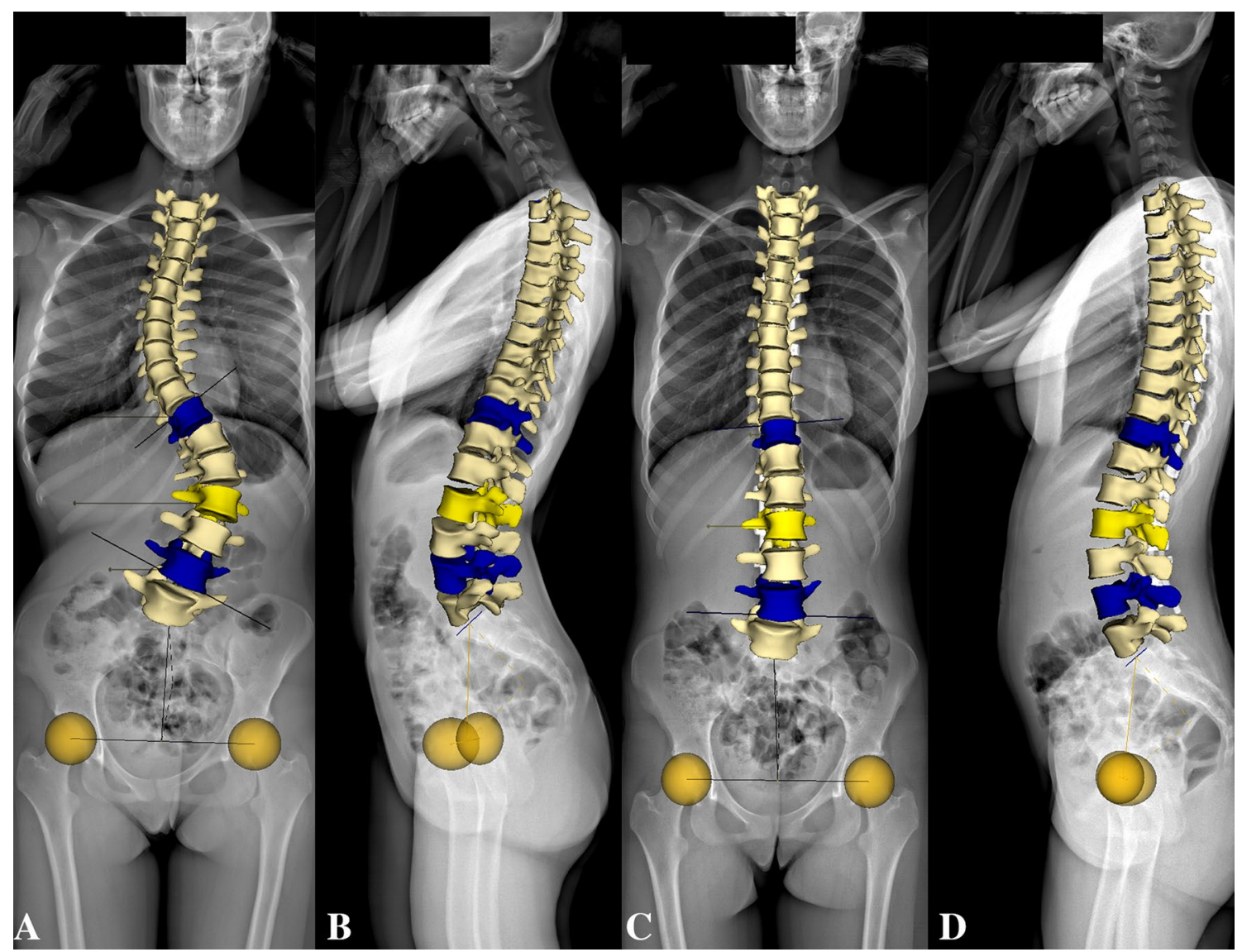

Fig. 3 Second case of scoliosis classified as Lenke 6/C. $53^{\circ}$ right thoracic and $61^{\circ}$ left lumbar main curves (a). The thoracic kyphosis between the T4 and T12 vertebrae is $6^{\circ}$; the lumbar lordosis between

immediately visible. The differences are even more pronounced after vertebral vector visualization (Fig. 4c, d). The postoperative $3 \mathrm{D}$ and vertebral vector top-view images for the first patient are shown in Fig. 4e-g, and those for the second patient are shown in Fig. $4 \mathrm{f}-\mathrm{h}$. The different coordinates have different meanings. The $x$-coordinates represent the relative distance of the respective points from $y$-axis, whereas the $y$ coordinates show the distance of the same points from the $x$-axis. The preoperative projected values of the start and end points of the vertebral vectors, their real distance from the $x$-axis and $y$-axis in mm, and the calculated projected axial rotations of the vertebrae of the first case of scoliosis are shown in Table 1 . The same preoperative data regarding the second case of scoliosis are shown in Table 2, whereas the postoperative data are presented in Tables 3 and 4 for the first and second cases of scoliosis, respectively.

In the first case before surgery, the vertebral vectors were arranged close to the $x$-axis (Fig. $4 \mathrm{a}-\mathrm{h}$ ). The $\mathrm{T} 1$ vertebra was displaced $\left(\mathrm{T}_{1} \mathrm{~A}_{x}=38.5\right) 29.7 \mathrm{~mm}$ to the left (Table 1: column 3, line 2) from the middle-sagittal axis of the body. The most lateralized vertebra to the left was the $\mathrm{L} 2(44.3 \mathrm{~mm})$ the L1 and L5 vertebrae is $47^{\circ}$ (b). The postoperative thoracic curve is $9^{\circ}$ and the lumbar curve is $6^{\circ}(\mathbf{c})$. The postoperative kyphosis increased to $27^{\circ}$, while the lordosis decreased to $42^{\circ}$ (d)

(Table 1: columns $2-3$, line 15), whereas the L5 vertebra was only lateralized $13.7 \mathrm{~mm}$ (Table 1: column 3 , line 18). The most axially rotated vertebra to the right was $\mathrm{T} 8$ (Table 1 : column 10, line 9), which was situated just in the middlesagittal axis ( $y$-axis) of the body (Table 1: column 2, line 9). The vertebra most rotated to the left was the $\mathrm{L} 1$ with $21.9^{\circ}$ of projected axial rotation (Table 1: column 10, line 14). The direction of projected axial rotation was changed the first time between T3 (Table 1: column 10, line 4) and Th4 (Table 1: column 10, line 5), and the second time between T11 (Table 1: column 10, line 12) and T12 (Table 1: column 10 , line 13).

However, in the second case before surgery, the vertebral vectors retained their position relative to the $x$-axis. The most lateralized vertebra to the right was the T7 vertebra (Table 3: columns 2-3, line 8) and that to the left was the L2 vertebra (Table 2: columns 2 and 3, line 15), which is located four times farther $(46.1 \mathrm{~mm})$ from the $y$-axis than the $\mathrm{T} 7$ vertebra $(-13.6 \mathrm{~mm})$. The $\mathrm{T} 1$ (Table 2: column 3 , line 2) and L5 (Table 2: column 3, line 18) vertebral vectors were situated at the same distance from the $y$-axis. The 

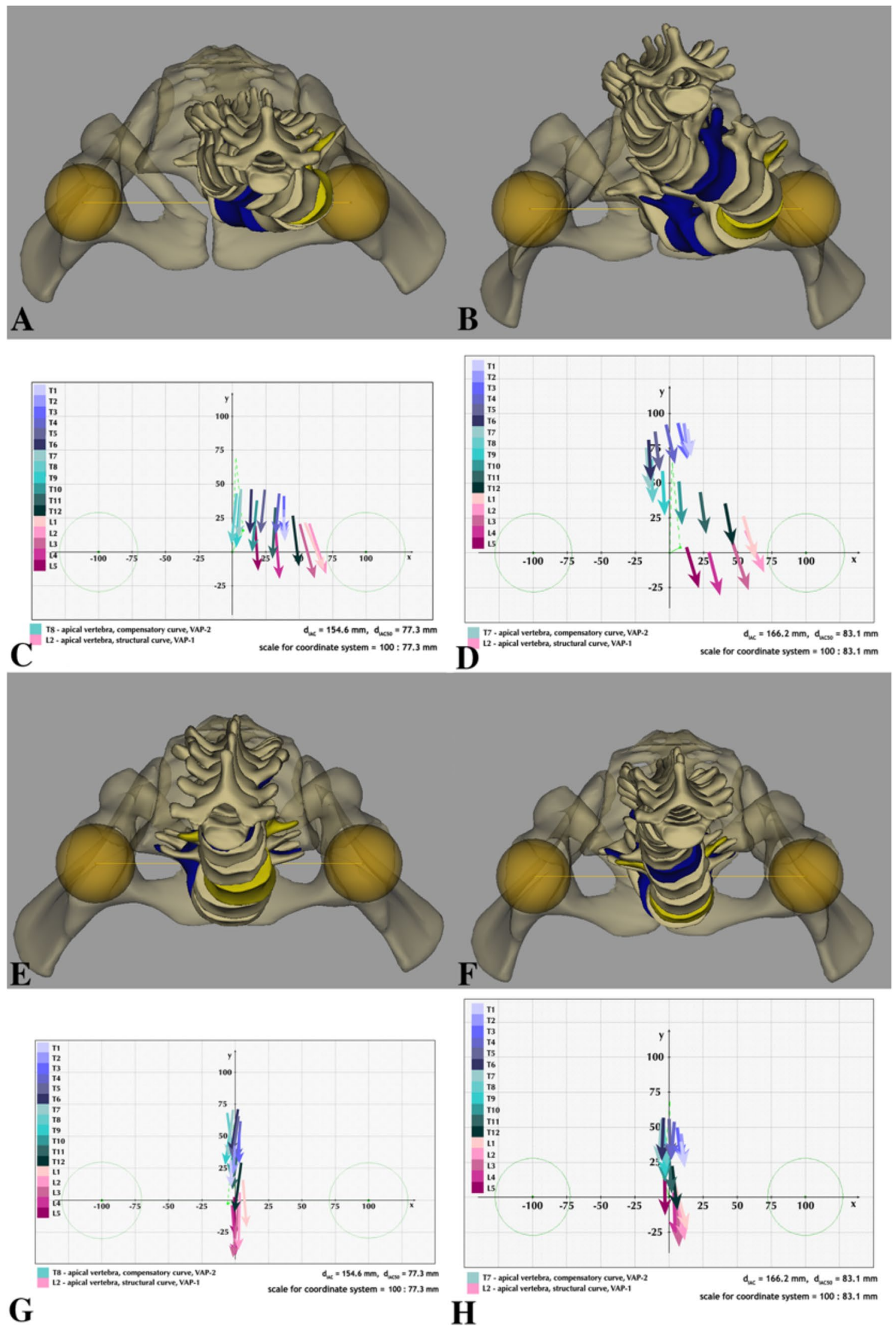
4Fig. 4 Three-dimensional (3D) and vertebral vector top-view visualization of both cases. Preoperative 3D top-view image of the first case of scoliosis (a). Preoperative 3D top-view image of the second case of scoliosis (b). Differences between the two curves are immediately visible. The differences are even more pronounced after vertebral vector visualization of the first (c) and second (d) cases of scoliosis. Postoperative 3D top-view image of the first case of scoliosis (e). Postoperative 3D top-view image of the second case of scoliosis (f). In the top view, only the trends of the corrections can be determined. Postoperative top-view image of the first case of scoliosis with the vertebral vector $(\mathbf{g})$. Postoperative top-view image of the second case of scoliosis with the vertebral vector (h). After visualizing the whole spine with vertebral vectors, the postoperative rearrangement of vertebrae became clearly visible and analyzable

most axially rotated vertebra to the right was T7 (Table 2: column 10 , line 8 ). The vertebra most rotated to the left was L2 with $25.7^{\circ}$ of projected axial rotation (Table 2: column 10 , line 15). The direction of projected axial rotation was changed only between $\mathrm{T} 7$ and $\mathrm{T} 8$, but it returned to the same direction as before between T8 and T9 (Table 2: column 10, lines 8-10).

After surgical correction, the top-view images became similar in both cases of scoliosis. The lateral displacements disappeared, and vertebrae aligned next to the $y$-axis, as can be seen from the vector coordinates comparing the preoperative and postoperative values (Tables 1, 2, 3, 4: columns 1-4).

In the first case, the $y$-coordinates of each thoracic vertebra increased considerably (Table 3: columns 6 and 8) compared to the preoperative value, which is the sign that the vertebrae had moved away from the $x$-axis (Table 3: columns 7 and 9). In the second case, the $y$-coordinates in thoracic vertebrae decreased (Table 4: columns 6 and 8 ) and the vertebrae approached the $x$-axis (Table 4: columns 7 and 9). In the lumbar area, the vertebral vector distances from the $x$-axis were not substantially changed (Table 4: columns 7 and 9). The projected axial rotations of each vertebra diminished (Tables 3 and 4: column 10) during surgery compared to the preoperative values (Tables 1 and 2: column 10).

\section{Discussion}

There is a great demand for visualizing the spinal curves in all three planes in patients with scoliosis, a 3D spinal deformity. The most accurate $3 \mathrm{D}$ visualization of the spine is based on the new EOS ${ }^{\mathrm{TM}} 2 \mathrm{D} / 3 \mathrm{D}$ radiographic images, allowing simultaneous full-body capturing of the upright perpendicular coronal and lateral radiographic images in a spatially calibrated way. The high-quality EOS ${ }^{\mathrm{TM}}$ images allow very accurate $3 \mathrm{D}$ surface reconstruction using sterEOS $^{\mathrm{TM}}$ 3D software $[15,16]$.

The 3D reconstruction software associated with EOS ${ }^{\mathrm{TM}}$ defines the coordinates in global anatomical landmarks from different points of vertebrae and deduces the coordinates of these vertebral marks. Using certain points of the aforementioned landmarks, we proposed a mathematical algorithm to define the posteroanterior vertebral axis, i.e., the vertebral vector. The vector is a mathematical entity characterized by its initial and terminal points by its length and spatial direction, commonly represented by a directed line segment. It is possible to visualize this vertebral vector (posteroanterior vertebral axis) projection on each of the planes and to determine the value of the projected vector angles relative to the anatomical axes in the planes on which the vectors are projected [13]. This method of representation also allows the evolution of vertebral vector projections in the axial plane before and after surgical treatment, along with representation of the entire spine.

The axial plane top-view image is essential to complete observation of the scoliosis curves, because, despite the similar representations in the frontal and sagittal planes, the occurrence of scoliosis in the horizontal plane can be entirely different, as it has been clearly shown in two patients demonstrated herein. The axial plane analysis together with evaluations of usual coronal and sagittal curves may reveal inherent structural differences that are not apparent in two planar radiographic assessments. Atmaca et al. reached the same conclusion after analyzing the axial plane changes of Lenke 1 curves using the Drerup method [17].

Thong et al. also found a difference in axial plane appearance [18] after evaluating the 3D sub-groups of all Lenke types of thoracic and lumbar scoliotic curves, although the methodology used (the PMC display) is only a quasi-3D spatial representation [10].

As the Lenke classification is already a lumbar and sagittal modifier, it seems necessary to also adjust the horizontal modifier.

In the first case, the whole spine was decompensated, because the T1 vertebra was displaced more laterally than the L5 vertebra. It was also clearly visible that the vertebrae were arranged near the $x$-axis. This is the sign of the sagittal curves' disappearance, resulting in a flat back. In the first case, the spine was not only decompensated, but it became flat too.

In contrast, the second patient did not have a decompensated spine, because the T1 and L5 vertebrae were situated at the same distance from the $y$-axis; hence, they were located above each other on the same plane. The sagittal curves did not decrease, because the vertebrae distances from the $x$-axis were constantly changing according to the necessity of the thoracic kyphosis and lumbar lordosis. The projected rotation of lumbar apical vertebrae was nearly the same: $22^{\circ}$ for the first patient and $25^{\circ}$ for the second patient.

After surgical correction, the top-view images became similar. The postoperative top-view image shows new realignment of the vertebrae in both patients. The vertebrae 
Table 1 Preoperative vector coordinates of the first case of scoliosis with the real distances calculated from the respective axis and the axial rotation of each vertebral vector

\begin{tabular}{lrrrrrrrrr}
\hline & \multicolumn{1}{c}{$A_{x}$} & $\mathrm{~mm}$ & \multicolumn{1}{c}{$B_{x}$} & $\mathrm{~mm}$ & $A_{y}$ & $\mathrm{~mm}$ & $B_{y}$ & $\mathrm{~mm}$ & $\circ$ \\
\hline $\mathrm{T} 1$ & 38.5 & 29.7 & 39.2 & 30.3 & 25.7 & 19.9 & 7.3 & 5.7 & 2.3 \\
$\mathrm{~T} 2$ & 38.5 & 29.8 & 38.6 & 29.9 & 31.7 & 24.5 & 11.2 & 8.6 & 0.3 \\
$\mathrm{~T} 3$ & 38.6 & 29.9 & 39.1 & 30.2 & 41.1 & 31.7 & 13.7 & 10.6 & 1.0 \\
$\mathrm{~T} 4$ & 35.1 & 27.1 & 32.7 & 25.2 & 43.1 & 33.3 & 13.0 & 10.0 & -4.5 \\
$\mathrm{~T} 5$ & 24.4 & 18.9 & 21.3 & 16.5 & 45.9 & 35.4 & 13.7 & 10.6 & -5.5 \\
$\mathrm{~T} 6$ & 14.1 & 10.9 & 12.0 & 9.3 & 46.3 & 35.8 & 13.1 & 10.1 & -3.6 \\
$\mathrm{~T} 7$ & 7.0 & 5.4 & 4.4 & 3.4 & 46.6 & 36.0 & 10.3 & 8.0 & -4.1 \\
$\mathrm{~T} 8$ & 3.8 & 2.9 & -1.2 & -1.0 & 43.4 & 33.5 & 7.3 & 5.6 & -7.9 \\
$\mathrm{~T} 9$ & 6.3 & 4.9 & 2.4 & 1.8 & 41.5 & 32.1 & 3.4 & 2.6 & -6.0 \\
$\mathrm{~T} 10$ & 18.0 & 13.9 & 14.3 & 11.0 & 38.2 & 29.5 & 0.3 & 0.3 & -5.7 \\
$\mathrm{~T} 11$ & 32.7 & 25.3 & 29.9 & 23.1 & 32.9 & 25.4 & -4.9 & -3.8 & -4.3 \\
$\mathrm{~T} 12$ & 45.9 & 35.5 & 50.3 & 38.9 & 26.9 & 20.8 & -10.8 & -8.3 & 6.7 \\
$\mathrm{~L} 1$ & 54.2 & 41.9 & 68.3 & 52.8 & 21.7 & 16.8 & -13.3 & -10.3 & 21.9 \\
$\mathrm{~L} 2$ & 57.4 & 44.3 & 70.3 & 54.3 & 21.2 & 16.4 & -15.6 & -12.1 & 19.3 \\
L3 & 50.2 & 38.8 & 62.1 & 48.0 & 20.9 & 16.2 & -19.4 & -15.0 & 16.4 \\
L4 & 32.9 & 25.4 & 36.8 & 28.5 & 18.3 & 14.2 & -20.0 & -15.5 & 5.9 \\
L5 & 17.7 & 13.7 & 19.0 & 14.7 & 17.9 & 13.9 & -10.3 & -7.9 & 2.6 \\
\hline
\end{tabular}

Table 2 Preoperative vector coordinates of the second case of scoliosis with the real distances calculated from the respective axis and the axial rotation of each vertebral vector

\begin{tabular}{lrrrrrrrrr}
\hline & \multicolumn{1}{c}{$A_{x}$} & $\mathrm{~mm}$ & \multicolumn{1}{c}{$B_{x}$} & \multicolumn{1}{c}{$\mathrm{mm}$} & \multicolumn{1}{c}{$A_{y}$} & $\mathrm{~mm}$ & \multicolumn{1}{c}{$B_{y}$} & $\mathrm{~mm}$ & $\circ$ \\
\hline $\mathrm{T} 1$ & -3.3 & -2.6 & -3.0 & -2.3 & 31.6 & 24.4 & 11.7 & 9.1 & 1.0 \\
$\mathrm{~T} 2$ & -0.2 & -0.2 & -0.8 & -0.6 & 43.1 & 33.3 & 18.8 & 14.5 & -1.4 \\
$\mathrm{~T} 3$ & 3.1 & 2.4 & 3.6 & 2.8 & 53.5 & 41.4 & 25.6 & 19.8 & 1.0 \\
$\mathrm{~T} 4$ & 3.9 & 3.0 & 1.9 & 1.4 & 62.2 & 48.1 & 31.6 & 24.4 & -3.9 \\
$\mathrm{~T} 5$ & 2.5 & 1.9 & -1.3 & -1.0 & 66.2 & 51.1 & 32.5 & 25.1 & -6.5 \\
$\mathrm{~T} 6$ & 2.1 & 1.6 & -3.5 & -2.7 & 71.4 & 55.2 & 36.6 & 28.3 & -9.0 \\
$\mathrm{~T} 7$ & -1.8 & -1.4 & -5.8 & -4.4 & 71.2 & 55.0 & 34.9 & 27.0 & -6.2 \\
$\mathrm{~T} 8$ & -5.8 & -4.5 & -1.7 & -1.3 & 68.8 & 53.2 & 31.7 & 24.5 & 6.3 \\
$\mathrm{~T} 9$ & -1.9 & -1.5 & -7.7 & -6.0 & 65.3 & 50.5 & 26.6 & 20.5 & -8.6 \\
$\mathrm{~T} 10$ & 1.1 & 0.8 & -3.8 & -2.9 & 53.2 & 41.1 & 17.8 & 13.8 & -7.8 \\
$\mathrm{~T} 11$ & 2.4 & 1.8 & -1.9 & -1.4 & 41.0 & 31.7 & 5.4 & 4.2 & -6.7 \\
$\mathrm{~T} 12$ & 4.6 & 3.6 & -0.5 & -0.4 & 29.3 & 22.7 & -10.2 & -7.9 & -7.3 \\
L1 & 6.0 & 4.6 & 9.0 & 7.0 & 16.4 & 12.6 & -24.6 & -19.0 & 4.3 \\
L2 & 3.3 & 2.6 & 2.4 & 1.9 & 6.5 & 5.0 & -35.4 & -27.4 & -1.2 \\
L3 & 2.5 & 1.9 & -1.4 & -1.1 & -3.2 & -2.4 & -44.0 & -34.0 & -5.4 \\
L4 & -2.1 & -1.6 & 0.6 & 0.4 & -3.5 & -2.7 & -43.2 & -33.4 & 3.8 \\
L5 & -0.5 & -0.4 & 0.1 & 0.1 & 3.3 & 2.5 & -29.8 & -23.0 & 1.1 \\
\hline & & & & & & & & &
\end{tabular}

regrouped close to the body axis. To obtain this new realignment of the spine, it seems to be more important to minimalize the lateral ejection of the spine and to move the vertebrae as close as possible to the body axis (in the top view from the $y$-axis) during the correction. To reduce the distance between the vertebrae and sagittal median axis of the body, the principal maneuver during the surgery has to be vertebral translation in the horizontal plane. This ascertainment is consistent with the observation of Seoud et al. in that the scoliosis reduction by spine translation provides good correction for the curves and for the rib humps [19].
In our opinion, reducing vertebral rotation is of secondary importance [20].

To translate the spine in the horizontal plane, it is not necessary to use screws in every segment, mainly in the thoracic area. Surgeons can obtain good translation also by performing instrumentation with hooks of strategic vertebrae, according to the Cotrel-Dubousset philosophy.

The use of only posteroanterior vertebral vectors in the horizontal plane provides different types of parameters regarding the impact of surgical treatment: reducing lateral deviations, achieving harmony of the curves in the sagittal 
Table 3 Postoperative vector coordinates of the first case of scoliosis with the real distances calculated from the respective axis and the axial rotation of each vertebral vector

\begin{tabular}{lrrrrrrrrr}
\hline & \multicolumn{1}{c}{$A_{x}$} & \multicolumn{1}{c}{$\mathrm{mm}$} & \multicolumn{1}{c}{$B_{x}$} & \multicolumn{1}{c}{$\mathrm{mm}$} & \multicolumn{1}{c}{$A_{y}$} & \multicolumn{1}{c}{$\mathrm{mm}$} & \multicolumn{1}{c}{$B_{y}$} & $\mathrm{~mm}$ & $\circ$ \\
\hline $\mathrm{T} 1$ & 13.0 & 10.8 & 15.8 & 13.1 & 89.1 & 74.0 & 69.3 & 57.6 & 8.0 \\
$\mathrm{~T} 2$ & 10.3 & 8.6 & 15.6 & 13.0 & 93.5 & 77.7 & 69.1 & 57.4 & 12.3 \\
$\mathrm{~T} 3$ & 6.0 & 5.0 & 13.5 & 11.2 & 93.7 & 77.9 & 67.6 & 56.1 & 15.9 \\
$\mathrm{~T} 4$ & -2.8 & -2.4 & 4.1 & 3.4 & 92.6 & 76.9 & 4.1 & 3.4 & 4.5 \\
$\mathrm{~T} 5$ & -10.8 & -9.0 & -7.9 & -6.6 & 87.4 & 72.6 & 57.7 & 48.0 & 5.6 \\
T6 & -15.4 & -12.8 & -14.6 & -12.2 & 81.7 & 67.9 & 51.9 & 43.1 & 1.5 \\
T7 & -16.4 & -13.6 & -15.0 & -12.5 & 76.3 & 63.4 & 45.7 & 37.9 & 2.5 \\
T8 & -13.0 & -10.8 & -13.5 & -11.2 & 68.1 & 56.6 & 37.5 & 31.2 & -0.8 \\
T9 & -5.8 & -4.8 & -5.0 & -4.1 & 59.4 & 49.3 & 27.9 & 23.2 & 1.6 \\
T10 & 6.5 & 5.4 & 8.5 & 7.0 & 51.5 & 42.8 & 20.5 & 17.0 & 3.7 \\
T11 & 22.8 & 18.9 & 27.4 & 22.8 & 43.8 & 36.4 & 13.3 & 11.1 & 8.7 \\
T12 & 41.0 & 34.1 & 47.4 & 39.4 & 35.8 & 29.7 & 4.9 & 4.1 & 11.6 \\
L1 & 53.7 & 44.6 & 63.3 & 52.6 & 26.6 & 22.1 & -4.4 & -3.6 & 17.2 \\
L2 & 55.5 & 46.1 & 69.7 & 57.9 & 16.5 & 13.7 & -13.0 & -10.8 & 25.7 \\
L3 & 46.7 & 38.8 & 59.4 & 49.3 & 8.1 & 6.7 & -26.4 & -22.0 & 20.1 \\
L4 & 29.2 & 24.2 & 36.9 & 30.6 & 0.6 & 0.5 & -30.8 & -25.6 & 13.8 \\
L5 & 11.6 & 9.7 & 18.8 & 15.6 & 4.7 & 3.9 & -23.3 & -19.4 & 14.2 \\
\hline
\end{tabular}

Table 4 Postoperative vector coordinates of the second case of scoliosis with the real distances calculated from the respective axis and the axial rotation of each vertebral vector

\begin{tabular}{lrrrrrrrrr}
\hline & \multicolumn{1}{c}{$A_{x}$} & $\mathrm{~mm}$ & \multicolumn{1}{c}{$B_{x}$} & \multicolumn{1}{c}{$\mathrm{mm}$} & \multicolumn{1}{c}{$A_{y}$} & $\mathrm{~mm}$ & \multicolumn{1}{c}{$B_{y}$} & $\mathrm{~mm}$ & $\circ$ \\
\hline $\mathrm{T} 1$ & 9.0 & 7.5 & 11.5 & 9.5 & 39.6 & 32.9 & 20.6 & 17.1 & 7.4 \\
$\mathrm{~T} 2$ & 8.9 & 7.4 & 11.2 & 9.3 & 45.4 & 37.8 & 21.7 & 18.0 & 5.6 \\
$\mathrm{~T} 3$ & 6.0 & 5.0 & 8.7 & 7.2 & 49.9 & 41.4 & 24.4 & 20.3 & 6.0 \\
$\mathrm{~T} 4$ & 4.2 & 3.5 & 2.1 & 1.7 & 53.9 & 44.8 & 26.4 & 22.0 & -4.3 \\
$\mathrm{~T} 5$ & 0.2 & 0.2 & 1.0 & 0.8 & 55.9 & 46.5 & 26.7 & 22.2 & 1.5 \\
$\mathrm{~T} 6$ & -4.2 & -3.4 & -5.8 & -4.8 & 56.7 & 47.1 & 25.4 & 21.1 & -3.0 \\
$\mathrm{~T} 7$ & -5.3 & -4.4 & -8.1 & -6.7 & 55.9 & 46.4 & 24.9 & 20.7 & -5.2 \\
$\mathrm{~T} 8$ & -4.5 & -3.8 & -1.7 & -1.4 & 53.8 & 44.7 & 21.8 & 18.1 & 5.0 \\
$\mathrm{~T} 9$ & -5.7 & -4.8 & -2.9 & -2.4 & 50.4 & 41.9 & 18.0 & 15.0 & 5.1 \\
$\mathrm{~T} 10$ & -3.8 & -3.1 & -2.2 & -1.8 & 43.4 & 36.0 & 11.0 & 9.1 & 2.8 \\
$\mathrm{~T} 11$ & -0.4 & -0.3 & 2.1 & 1.7 & 33.7 & 28.0 & 2.4 & 2.0 & 4.5 \\
$\mathrm{~T} 12$ & 2.5 & 2.0 & 6.6 & 5.4 & 22.1 & 18.3 & -10.4 & -8.6 & 7.2 \\
L1 & 3.8 & 3.2 & 13.5 & 11.2 & 11.4 & 9.5 & -22.4 & -18.6 & 16.0 \\
L2 & 5.2 & 4.3 & 12.5 & 10.4 & 3.9 & 3.3 & -30.8 & -25.6 & 11.8 \\
L3 & 6.3 & 5.2 & 7.9 & 6.6 & -1.0 & -0.8 & -36.6 & -30.4 & 2.7 \\
L4 & 3.7 & 3.1 & 4.6 & 3.9 & 3.2 & 2.7 & -29.6 & -24.6 & 1.6 \\
L5 & -3.0 & -2.5 & -2.8 & -2.4 & 13.4 & 11.1 & -13.7 & -11.4 & 0.4 \\
\hline
\end{tabular}

plane, and reducing rotations in the horizontal plane. We can obtain information from all three planes of the spine in one image [21].

This view uniquely allows a good assessment of the 3D situation of the spine and provides useful information in daily clinical practice before and after surgical treatment; furthermore, it facilitates the understanding of the $3 \mathrm{D}$ nature of scoliosis. Implementation of this approach is simple. These results are sufficient for visual analysis, exhibiting a significant amount of clinical information in the three anatomical planes. This visualization approach represents a reasonable compromise between mathematical purity and practical use.

Acknowledgements We acknowledge Editage for language editing.

Funding There is no funding source.

\section{Compliance with ethical standards}

Conflict of interest The authors declare that they have no conflict of interest. 
Ethical standards All procedures performed in studies involving human participants were in accordance with the ethical standards of the institutional and/or national research committee and with the 1964 Helsinki declaration and its later amendments or comparable ethical standards.

Informal consent For this type of study, formal consent is not required.

\section{References}

1. Graf H, Hecquet J, Dubousset J (1983) 3-Dimensional approach to spinal deformities. Application to the study of the prognosis of pediatric scoliosis. Rev Chir Orthop Reparatrice Appar Mot 69:407-416

2. De Smet AA, Tarlton MA, Cook LT, Berridge AS, Asher MA (1983) The top view for analysis of scoliosis progression. Radiology 2:369-372. https://doi.org/10.1148/radiology.147.2.6340156

3. Howell FR, Dickson RA (1989) The deformity of idiopathic scoliosis made visible by computer graphics. J Bone Jt Surg Br 71:399-403

4. Vrtovec T, Franjo Pernus F, Likar B (2009) A review of methods for quantitative evaluation of axial vertebral rotation. Eur Spine $\mathrm{J}$ 18:1079-1090. https://doi.org/10.1007/s00586-009-0914-z

5. Drerup B (1984) Improvements in measuring vertebral rotation from the projections of the pedicles. J Biomech 18:369-378

6. Gardner-Morse M, Stokes IA (1994) Three-dimensional simulations of the scoliosis derotation maneuver with Cotrel-Dubousset instrumentation. J Biomech 27:177-181

7. Kojima T, Kurokawa T (1992) Rotation vector, a new method for representation of three-dimensional deformity in scoliosis. Spine (Phila Pa 1976) 17:1296-1303

8. Stokes IA, Gardner-Morse M (1993) Three-dimensional simulation of Harrington distraction instrumentation for surgical correction of scoliosis. Spine (Phila Pa 1976) 18:2457-2464

9. Stagnara P, Queneau P (1953) Scolioses évolutives en période de croissance: aspects cliniques, radiologiques, propositions thérapeutiques. Rev Chir Orthop 39:378-449

10. Stokes IA (1994) Three-dimensional terminology of spinal deformity. A report presented to the Scoliosis Research Society by the Scoliosis Research Society Working Group on 3-D terminology of spinal deformity. Spine (Phila Pa 1976) 19:236-248

11. Yazici M, Acaroglu ER, Alanay A, Deviren V, Cila A, Surat A (2001) Measurement of vertebral rotation in standing versus supine position in adolescent idiopathic scoliosis. J Pediatr Orthop 21:252-256

12. Lenke LG, Betz RR, Harms J, Bridwell KH, Clements DH, Lowe TG, Blanke K (2001) Adolescent idiopathic scoliosis. A new classification to determine extent of spinal arthrodesis. J Bone Jt Surg Am 83-A:1169-1181

13. Illés T, Tunyogi-Csapó M, Sz Somoskeőy (2011) Breakthrough in three-dimensional scoliosis diagnosis: significance of horizontal plane view and vertebra vectors. Eur Spine J 20:135-143. https:// doi.org/10.1007/s00586-010-1566-8

14. Dubousset J, Charpak G, Dorion I, Skalli W, Lavaste F, Deguise J, Kalifa G, Ferey S (2005) A new 2D and 3D imaging approach to musculoskeletal physiology and pathology with low-dose radiation and the standing position: the EOS system. Bull Acad Natl Med 189:287-297

15. Le Bras A, Laporte S, Mitton D, de Guise JA, Skalli W (2002) 3D detailed reconstruction of vertebrae with low dose digital stereoradiography. Stud Health Technol Inform 91:286-290

16. Laporte S, Skalli W, de Guise JA, Lavaste F, Mitton D (2003) A biplanar reconstruction method based on $2 \mathrm{D}$ and $3 \mathrm{D}$ contours: application to the distal femur. Comput Methods Biomech Biomed Engin 6:1-6. https://doi.org/10.1080/1025584031000065956

17. Atmaca H, Inanmaz ME, Bal E, Caliskan I, Kose KC (2014) Axial plane analysis of Lenke 1A adolescent idiopathic scoliosis as an aid to identify curve characteristics. Spine J 14:2425-2433. https ://doi.org/10.1016/j.spinee.2014.02.015

18. Thong W, Parent S, Wu J, Aubin C-E, Labelle H, Kadoury S (2016) Three-dimensional morphology study of surgical adolescent idiopathic scoliosis patient from encoded geometric models. Eur Spine J 25:3104-3113. https://doi.org/10.1007/s0058 6-016-4426-3

19. Seoud L, Cheriet F, Labelle H, Parent S (2015) Changes in trunk appearance after scoliosis spinal surgery and their relation to changes in spinal measurements. Spine Deform 3:595-603. https ://doi.org/10.1016/j.jspd.2015.05.001

20. Illés TS, Somoskeôy S (2013) Comparison of scoliosis measurements based on three-dimensional vertebra vectors and conventional two-dimensional measurements: advantages in evaluation of prognosis and surgical results. Eur Spine J 22:1255-1263. https ://doi.org/10.1007/s00586-012-2651-y

21. Illés ST, Burkus M, Somoskeőy S, Lauer F, Lavaste F, Dubousset JF (2017) The horizontal plane appearances of scoliosis: what information can be obtained from top-view images? Int Orthop 41:2303-2311. https://doi.org/10.1007/s0026 